\title{
ORIGINAL
}

\section{EL COSTE DE VACUNAR A LO LARGO DE TODA LA VIDA EN ESPAÑA}

Marta Soler Soneira (1), Carmen Olmedo Lucerón (1), Laura Sánchez-Cambronero Cejudo (1), Elena Cantero Gudino (2)

y Aurora Limia Sánchez (1)

(1) Área de Programas de Vacunación. Subdirección General de Promoción de la Salud y Vigilancia en Salud Pública. Dirección General de Salud Pública, Calidad e Innovación. Ministerio de Sanidad, Consumo y Bienestar Social. Madrid. España.

(2) Asistencia técnica TRAGSATEC en el Ministerio de Sanidad, Consumo y Bienestar Social. Madrid. España.

Las autoras declaran que no existe conflicto de intereses.

\section{RESUMEN}

Fundamentos: La evaluación de los programas de vacunación mediante la estimación de costes es una herramienta fundamental para orientar la política de vacunación. El objetivo de este trabajo fue describir el coste que conlleva en España la vacunación a lo largo de toda la vida, tanto a personas sanas como pertenecientes a grupos de riesgo.

Métodos: Se realizó un estudio de descripción de los costes para administrar las vacunas incluidas en el calendario común de vacunación acordado para el año 2019, y en el calendario para grupos de riesgo, a lo largo de toda la vida.

Resultados: El coste previsto de la vacunación a lo largo de toda la vida fue de 726,06 euros por cada mujer sana y 625,89 euros por cada hombre sano durante el 2019. En personas con las condiciones de riesgo que requieren mayor número de vacunas osciló entre 982,99 y 1.815 euros por persona.

Conclusiones: El relativo bajo coste de la vacunación a lo largo de toda la vida y los importantes beneficios para la salud que conlleva la vacunación hacen que esta medida sea útil y rentable, por lo que se debe reforzar la evaluación de los programas de vacunación para asegurar la vacunación adecuada en todos los momentos de la vida.

Palabras clave: Programas de vacunación, Vacunas, Evaluación, Costes, Grupos de Riesgo.

\section{ABSTRACT The cost of vaccination throughout life in Spain}

Background: The evaluation of vaccination programmes using cost estimation is an essential tool for immunization policy. The aim of this study was to describe the cost of vaccination throughout life in Spain, both in healthy and risk groups persons.

Methods: Description of cost of vaccination following the national immunization programme throughout life agreed for 2019, and the immunization programme for risk groups.

Results: The expected cost to immunize a healthy person is 726.06 euros for a healthy woman and 625.89 euros for a healthy man, ranging from 982.99 to 1,815 euros per person in risk groups.

Conclusions: The relatively low cost and the important benefits for health of immunization throughout life make this public health measure useful and worthwhile. Evaluation of immunization programmes should be strengthened in order to assure suitable immunization in every stage of life.

Key words: Vaccination programmes, Vaccines, Evaluation, Costs, Risk groups.

\footnotetext{
Cita sugerida: Soler Soneira M, Olmedo Lucerón C, SánchezCambronero Cejudo L, Cantero Gudino E, Limia Sánchez A. El coste de vacunar a lo largo de toda la vida en España. Rev Esp Salud Pública. 2020; 94: 11 de febrero e202002005
} 


\section{INTRODUCCIÓN}

La vacunación es una de las medidas de salud pública que más ha contribuido a disminuir la incidencia de enfermedades transmisibles. Actualmente, más de 25 enfermedades son prevenibles mediante vacunación. Además de la erradicación de la viruela, se han conseguido grandes avances hacia la erradicación de la poliomielitis y la eliminación del sarampión y la rubeola, así como en el control de otras enfermedades inmunoprevenibles ${ }^{(1)}$.

La incorporación de vacunas en el calendario sistemático ha ido modificando la epidemiología de las enfermedades prevenibles por vacunación hasta reducir a mínimos históricos su impacto en la morbilidad, la mortalidad y en los costes sanitarios asociados ${ }^{(2)}$. La implantación de los programas de vacunación supone un gran esfuerzo de recursos y logística, por lo que es necesario realizar evaluaciones que aseguren su adecuado funcionamiento, estableciendo medidas de mejora si se considera oportuno ${ }^{(3)}$.

En el momento actual, España dispone de un calendario de vacunación a lo largo de toda la vida acordado para el año 2019, que se actualizó en marzo de 2019 con la incorporación de la vacuna frente a meningococo de serogrupos A, C, W e Y (MenACWY) ${ }^{(4)}$.

Las herramientas de estimación de costes son esenciales para la planificación, gestión, evaluación correcta y la mejora continuada de los programas de vacunación ${ }^{(5)}$. Sin embargo, aunque existen numerosos estudios de análisis de costes como paso previo a la introducción de nuevas vacunas en el calendario común ${ }^{(6)}$, existen pocos trabajos que recojan de forma integrada la información sobre el coste que supone la vacunación de una persona durante toda la vida ${ }^{(7)}$. Disponer de esta información puede ser un paso previo a un análisis de coste-beneficio más exhaustivo y servir para comparar alternativas en prevención.
El objetivo de este trabajo fue describir el coste que conlleva la vacunación a lo largo de toda la vida en España, tanto a personas sanas como a personas pertenecientes a grupos de riesgo.

\section{MATERIAL Y MÉTODOS}

Se realizó un estudio de descripción de costes. Estos estudios no comparan alternativas ni evalúan resultados, pero son útiles como una primera valoración económica ${ }^{(8,9)}$. Se incluyeron los costes directos de la vacunación (vacuna y administración de la misma).

Vacunas consideradas. Tomando como referencia la definición de adulto sano realizada en las recomendaciones de vacunación en población adulta $^{(9)}$, desde el punto de vista de la vacunación se considera persona sana a toda persona que no tiene condiciones de riesgo que requiera la administración de una vacunación específica. Para estimar el cálculo del coste de la vacunación en personas sanas se consideraron las vacunas incluidas en el calendario común de vacunación a lo largo de toda la vida, acordado en el Consejo Interterritorial del Sistema Nacional de Salud para el año 2019, actualizado en marzo de 2019 con la introducción de la vacuna de meningococo ACWY (tabla 1). Para estimar el coste de vacunar a las personas pertenecientes a grupos de riesgo se consideraron las vacunas incluidas en el calendario para estos grupos ${ }^{(10)}$.

Suposiciones. Los cálculos se efectuaron atendiendo a las siguientes premisas:

i) La estimación se realizó considerando el calendario vigente para 2019, tras la actualización realizada en marzo, y para las características de la población general española.

ii) Se consideró un coste de la administración de 6 euros, independientemente del número de vacunas administradas en una misma visita. Es decir, si se administraban 3 vacunas en una 


\section{Tabla 1}

Enfermedades prevenibles por vacunación incluidas en el calendario común de vacunación a lo largo de toda la vida para 2019.

\begin{tabular}{|c|c|c|c|}
\hline \multirow{2}{*}{\multicolumn{2}{|c|}{ Enfermedad }} & \multicolumn{2}{|c|}{ Población } \\
\hline & & $\begin{array}{l}\text { Persona } \\
\text { sana }\end{array}$ & $\begin{array}{c}\text { Grupo } \\
\text { de riesgo }\end{array}$ \\
\hline \multicolumn{2}{|c|}{ Poliomielitis (VPI) } & $\checkmark$ & $\checkmark$ \\
\hline Difteria & \multirow{3}{*}{ DTPa } & $\checkmark$ & $\checkmark$ \\
\hline Tétanos & & $\checkmark$ & $\checkmark$ \\
\hline Tos ferina & & $\checkmark$ & $\checkmark$ \\
\hline \multicolumn{2}{|c|}{$\begin{array}{l}\text { Haemophilus } \\
\text { influenzae b (Hib) }\end{array}$} & $\checkmark$ & $\checkmark$ \\
\hline \multicolumn{2}{|l|}{ Sarampión } & $\checkmark$ & $\checkmark$ \\
\hline \multicolumn{2}{|l|}{ Rubéola } & $\checkmark$ & $\checkmark$ \\
\hline \multicolumn{2}{|l|}{ Parotiditis } & $\checkmark$ & $\checkmark$ \\
\hline \multicolumn{2}{|c|}{ Hepatitis B } & $\checkmark$ & $\checkmark$ \\
\hline \multicolumn{2}{|l|}{$\mathbf{E M I}^{(*)} \mathbf{C}$} & $\checkmark$ & $\checkmark$ \\
\hline \multicolumn{2}{|c|}{$\mathbf{E M I}^{(*)} \mathrm{ACWY}$} & $\checkmark$ & $\checkmark$ \\
\hline \multicolumn{2}{|l|}{$\mathbf{E M I}^{(*)} \mathbf{B}$} & $x$ & $\checkmark$ \\
\hline \multicolumn{2}{|l|}{ Varicela } & $\checkmark$ & $\checkmark$ \\
\hline \multicolumn{2}{|l|}{$\mathbf{V P H}^{(* *)}$} & $\begin{array}{l}\text { Sólo niñas } \\
\text { o mujeres }\end{array}$ & $\checkmark$ \\
\hline \multicolumn{2}{|c|}{$\begin{array}{l}\text { Enfermedad } \\
\text { neumocócica }\end{array}$} & $\checkmark$ & $\checkmark$ \\
\hline \multicolumn{2}{|l|}{ Gripe } & $\begin{array}{c}\text { A partir de } \\
65 \text { años }\end{array}$ & $\checkmark$ \\
\hline
\end{tabular}

(*) Enfermedad meningocócica invasora; (**) Virus del Papiloma Humano.

misma visita, el coste de la administración de todas ellas era un total de 6 euros. Esta cifra se obtuvo a partir de estudios de coste-efectividad que utilizaron como fuente las tarifas a partir de los Boletines Oficiales de las comunidades autónomas $^{(11)}$ y de estudios que estiman el tiempo de consulta de enfermería y resto de costes directos asociados a la administración de una vacuna ${ }^{(12)}$.

iii) Se asumió que las embarazadas recibieron la vacuna dTpa y de gripe en distintas visitas, aunque pudieran coincidir en el tiempo.

iv)Se supuso que tanto la mujer como el hombre fueron vacunados en la infancia con dos dosis de vacuna frente a la varicela (a los 15 meses y entre los tres y cuatro años) y, por tanto, no se tuvo en cuenta en el cálculo la vacunación frente a la varicela a los 12 años.

v) La vacuna combinada DTPa/IPV no se encontraba disponible en el momento del estudio y por tanto no tenía precio asignado. Se tomó como referencia el precio de la vacuna hexavalente. Esta vacunación se incluyó en el calendario porque se aprobó en el año 2017 para la población infantil que alcanzaba los 6 años y que se habían vacunado anteriormente con vacuna hexavalente mediante pauta $2+1$. Aunque estos niños y niñas todavía no habían alcanzado los 6 años y por tanto aún no recibían esta vacunación, se consideraron para tener un cálculo de máximos.

vi) Sin embargo, no se añadieron los costes de vacunación de Hepatitis B (HB) al nacimiento, asumiendo que no había nacimientos de madre portadora. Actualmente, debido a las coberturas elevadas de vacunación desde la implantación de la vacunación frente a la $\mathrm{HB}$, esta vacunación se realiza en muy pocas ocasiones.

vii) Dado el gran número y variabilidad de las condiciones consideradas en los grupos de riesgo, la estimación se realizó en aquellos que requerían mayor número de vacunas $\mathrm{y}$, por tanto, mayor gasto por persona. Estos fueron: "inmunodepresión (no VIH)", "VIH con CD4>200", "asplenia, deficiencias del complemento y tratamiento con eculizumab", 
"enfermedad renal crónica avanzada y hemodiálisis", "enfermedad hepática crónica", "trasplante de órgano sólido" y "TPH o trasplante de progenitores hematopoyéticos". En los grupos de riesgo considerados, se asumió que la condición o patología de riesgo estaba presente al nacimiento y tenían la misma esperanza de vida que las personas sanas, para obtener así el coste máximo de vacunación por grupo.

viii) Así, para el cálculo del número de vacunas de gripe que se administraban a lo largo de la vida en personas sanas, se tuvo en cuenta que la esperanza de vida media en nuestro país es de 83 años $^{(13)}$ :

- En personas sanas se estimaron 18 dosis de gripe a lo largo de toda la vida, teniendo en cuenta que la recomendación de la vacuna frente a la gripe es la administración a partir de los 65 años.

- En los grupos de riesgo considerados, se estimaron 83 dosis de gripe a lo largo de toda la vida. En VIH (CD4>200) se consideró que la infección se produjo concretamente al comienzo de tener relaciones sexuales (16 años; 67 dosis de gripe). Sin embargo, en las enfermedades crónicas y trasplante de órganos valoradas, aunque existía una enorme variabilidad en el momento del diagnóstico y en la esperanza de vida, solían aparecer en la edad media de la vida y reducir considerablemente su esperan$\mathrm{za}^{(14,15,16)}$. Así, se consideró que la edad media al diagnóstico fue de 50 años, con una esperanza de vida media de 20 años (se administrarían 20 dosis de gripe).

ix)En los grupos de riesgo cuyas condiciones o patologías de riesgo se conseguían en la edad media, se asumió que la condición se adquiría a los 50 años, con una esperanza de vida de 20 años a partir de ese momento. Así, se tuvieron en cuenta las vacunas administradas a una persona sana, añadiendo las vacunas recomendadas para la condición de riesgo adquirida que recibía posteriormente.

x) No se incluyó la vacuna inactivada frente a herpes zoster recomendada en algunos grupos de riesgo ${ }^{(11)}$, ya que por el momento no está disponible en nuestro país $\mathrm{y}$, por tanto, no tiene un precio establecido.

xi) Para el cálculo total de vacunar a lo largo de toda la vida se consideraron a los nacidos en 2018, a partir de la información disponible de nacimientos en 2018 del Instituto Nacional de Estadística (INE), estimando el coste de vacunación por sexo. Se estimó que las coberturas de vacunación alcanzadas serían del 100\%.

Precios. Se tuvieron en cuenta los precios fijados para cada vacuna en el Acuerdo Marco para la selección de suministradores de vacunas de calendario para el periodo 2017-2019(17) y en el Acuerdo Marco para la selección de suministradores de vacunas de gripe estacional ${ }^{(18)}$. Se examinaron los precios a fecha de 17 de abril de 2019, sin considerar el IVA. La vacuna conjugada frente a neumococo y la vacuna frente a meningococo B no estaban incluidas dentro del Acuerdo Marco, y se utilizó el precio de estas vacunas en los contratos de las comunidades autónomas de Murcia y Aragón.

Finalmente, para facilitar la comprensión de los resultados, se incorporó "en el siguiente apartado" o en "Resultados" la información referente al número estimado de personas en España que presentaban cada una de las condiciones de riesgo consideradas $^{(9,13,19,20,21,22,23,24,25)}$.

\section{RESULTADOS}

Se obtuvieron resultados para personas sanas y para personas pertenecientes a grupos de riesgo, además del coste de vacunar a lo largo de toda la vida. 
Personas sanas. El coste previsto en la vacunación a lo largo de toda la vida de una persona sana fue de 726,06 euros por cada mujer sana y 625,89 euros por cada hombre sano (tabla 2). Este coste se debió mayoritariamente a la vacunación en la etapa infantil y adolescente (485,90 euros en cada mujer sana y 421,58 euros en cada hombre sano).

El 25\% de este coste se correspondió con la administración de las vacunas (186 euros en las mujeres y 162 euros en el caso de los hombres).

Grupos de riesgo. Se seleccionaron y evaluaron los siguientes grupos de riesgo (tablas 2 a 5):

- VIH con CD4>200: durante el año 2016 se notificaron en España 3.353 nuevos diagnósticos de VIH (con una mediana edad de 36 años). A lo largo de toda la vida, el gasto estimado en la vacunación de una persona VIH con CD4>200 fue de 1.469,72 euros.

- Inmunodepresión (no VIH): se consideró como ejemplo el padecimiento de inmunodeficiencia primaria. Entre 1993 y 2001 se registraron 2.050 casos de inmunodeficiencias primarias. A lo largo de toda la vida, el gasto estimado en la vacunación de una persona con inmunodepresión (no VIH) fue de 1.491,69 euros.

- Asplenia, deficiencias del complemento y tratamiento con eculizumab: en España se realizan un promedio anual de 3.000 esplenectomías anuales. El 10\% de las inmunodeficiencias primarias eran deficiencias del sistema del complemento. En 2010, 50.000 personas se encontraban en tratamiento con agentes biológicos. A lo largo de toda la vida, el gasto estimado en la vacunación de una persona perteneciente a este grupo fue de 1.815 euros.

- Enfermedad renal crónica avanzada y hemodiálisis: según los resultados del estudio

\section{Tabla 2}

Coste de vacunar a lo largo de toda la vida en personas sanas, según edad, vacuna y sexo.

\begin{tabular}{|c|c|c|c|c|}
\hline $\begin{array}{l}\text { Grupo } \\
\text { edad }\end{array}$ & \multicolumn{2}{|r|}{ Vacuna } & $\begin{array}{l}\text { Precio } \\
\text { Mujer }^{(*)}\end{array}$ & $\begin{array}{c}\text { Precio } \\
\text { Hombre }^{(*)}\end{array}$ \\
\hline \multirow{4}{*}{$\begin{array}{l}\text { Pre- } \\
\text { natal }\end{array}$} & \multicolumn{2}{|c|}{ dTpa embarazada } & 14,95 & - \\
\hline & \multicolumn{2}{|c|}{$\begin{array}{l}\text { Administración } \\
\text { dTpa embarazada }\end{array}$} & 6,00 & - \\
\hline & \multicolumn{2}{|c|}{ Gripe no adyuvada } & 2,90 & - \\
\hline & \multicolumn{2}{|c|}{$\begin{array}{l}\text { Administración } \\
\text { gripe no adyuvada }\end{array}$} & 6,00 & - \\
\hline \multirow{3}{*}{$\begin{array}{l}2 \\
\text { meses }\end{array}$} & \multicolumn{2}{|c|}{ Hexavalente } & 29,95 & 29,95 \\
\hline & \multicolumn{2}{|c|}{ Neumococo 13} & 42,69 & 42,69 \\
\hline & \multicolumn{2}{|c|}{ Administración } & 6,00 & 6,00 \\
\hline \multirow{4}{*}{$\begin{array}{l}4 \\
\text { meses }\end{array}$} & \multicolumn{2}{|c|}{ Hexavalente } & 29,95 & 29,95 \\
\hline & \multicolumn{2}{|c|}{ Meningococo C } & 14,45 & 14,45 \\
\hline & \multicolumn{2}{|c|}{ Neumococo 13} & 42,69 & 42,69 \\
\hline & \multicolumn{2}{|c|}{ Administración } & 6,00 & 6,00 \\
\hline \multirow{3}{*}{$\begin{array}{l}11 \\
\text { meses }\end{array}$} & \multicolumn{2}{|c|}{ Hexavalente } & 29,95 & 29,95 \\
\hline & \multicolumn{2}{|c|}{ Neumococo 13} & 42,69 & 42,69 \\
\hline & \multicolumn{2}{|c|}{ Administración } & 6,00 & 6,00 \\
\hline \multirow{3}{*}{$\begin{array}{l}12 \\
\text { meses }\end{array}$} & \multicolumn{2}{|c|}{ Triple vírica } & 7,10 & 7,10 \\
\hline & \multicolumn{2}{|c|}{ Meningococo $\mathrm{C}$} & 14,45 & 14,45 \\
\hline & \multicolumn{2}{|c|}{ Administración } & 6,00 & 6,00 \\
\hline \multirow{2}{*}{$\begin{array}{l}15 \\
\text { meses }\end{array}$} & \multicolumn{2}{|c|}{ VVZ } & 19,95 & 19,95 \\
\hline & Admini & stración & 6,00 & 6,00 \\
\hline & Triple v & írica & 7,10 & 7,10 \\
\hline $\begin{array}{l}3-4 \\
\text { 3-n }\end{array}$ & Varicela & & 19,95 & 19,95 \\
\hline & Admini & stración & 6,00 & 6,00 \\
\hline 6 & DTPa II & $\mathrm{PV}^{(* *)}$ & 29,95 & 29,95 \\
\hline años & Admini & stración & 6,00 & 6,00 \\
\hline & Mening & ogoco ACWY & 32,30 & 32,30 \\
\hline & & VPH (niñas) & 29,16 & - \\
\hline 12 & Varicela & Administración & 6,00 & - \\
\hline & & VPH (niñas) & 29,16 & - \\
\hline & & Administración & 6,00 & - \\
\hline 14 & $\mathrm{Td}$ & & 4,41 & 4,41 \\
\hline años & Admini & stración & 6,00 & 6,00 \\
\hline & $\mathrm{Td}$ & & 4,41 & 4,41 \\
\hline$>65$ & Gripe a & dyuvada & 4,30 & 4,30 \\
\hline años & Neumo & $\operatorname{coco} 23$ & 10,20 & 10,20 \\
\hline & Admini & stración & 6,00 & 6,00 \\
\hline Gripe & Gripe a & dyuvada & 77,40 & 77,40 \\
\hline & Admini & stración & 108,00 & 108,00 \\
\hline Gasto & admini & stración & 186 & 162 \\
\hline$\%$ & & & 25,62 & 25,88 \\
\hline TOTA & & & 726,06 & 625,89 \\
\hline
\end{tabular}

(*) Precio en euros; (**) Precio de hexavalente, vacuna tetravalente todavía no disponible; $(* * *)$ Personas que refieran no haber pasado la enfermedad ni haber sido vacunadas con anterioridad. Pauta con 2 dosis; (****) Cálculo desde los 65 años teniendo en cuenta la esperanza de vida media de 83 años (18 dosis). 


\section{Tabla 3}

Coste de vacunar a lo largo de toda la vida en grupos de riesgo según vacuna: alteraciones inmunológicas.

\begin{tabular}{|c|c|c|c|}
\hline $\begin{array}{l}\text { Grupo } \\
\text { de riesgo }\end{array}$ & Vacunas & $\begin{array}{c}\text { Precio } \\
\text { dosis }\end{array}$ & $\begin{array}{c}\text { Coste final } \\
\text { con adminis- } \\
\text { tración }\end{array}$ \\
\hline \multirow{5}{*}{$\begin{array}{l}\text { Inmunode- } \\
\text { presión } \\
\text { (no VIH) }\end{array}$} & Gripe anual & 2,90 & 738,70 \\
\hline & $\begin{array}{l}\text { Neumococo } 23 \\
\text { (dosis recuerdo } \\
\text { a los } 65 \text { años) }\end{array}$ & 10,2 & 16,2 \\
\hline & $\begin{array}{l}\text { Hepatitis A } \\
\text { (si riesgo alto } \\
\text { de exposición) }\end{array}$ & 29,95 & 71,90 \\
\hline & $\begin{array}{l}\text { Vacunas del } \\
\text { calendario } \\
\text { (sin TV } \\
\text { y VVZ) }^{(* *)}\end{array}$ & - & 642,92 \\
\hline & \multicolumn{2}{|l|}{ TOTAL } & $1.469,72$ \\
\hline \multirow{8}{*}{$\begin{array}{l}\text { VIH } \\
(\text { CD4 } \\
>200) \\
\left({ }^{*}\right)\end{array}$} & Men ACWY & 32,30 & 114,90 \\
\hline & VPH & 29,16 & 105,48 \\
\hline & Hepatitis A & 29,95 & 71,90 \\
\hline & Gripe anual & 2,90 & 596,30 \\
\hline & Neumococo 13 & 42,69 & 48,69 \\
\hline & Neumococo 23 & 10,20 & 16,20 \\
\hline & $\begin{array}{l}\text { Vacunas } \\
\text { calendario }^{(* * *)}\end{array}$ & - & 675,97 \\
\hline & \multicolumn{2}{|l|}{ TOTAL } & $1.629,44$ \\
\hline \multirow{8}{*}{$\begin{array}{l}\text { Asplenia, } \\
\text { deficiencias } \\
\text { complemento } \\
\text { y tratamiento } \\
\text { con } \\
\text { eculizumab }\end{array}$} & Hib & 7,69 & 13,69 \\
\hline & $\begin{array}{l}\text { Meningococo } \\
\text { ACWY }\end{array}$ & 32,30 & 114,90 \\
\hline & Meningococo B & 62,95 & 206,85 \\
\hline & Gripe anual & 2,90 & 738,70 \\
\hline & Neumococo 13 & 42,69 & 48,69 \\
\hline & Neumococo 23 & 10,2 & 16,2 \\
\hline & $\begin{array}{l}\text { Vacunas de } \\
\text { calendario }^{(* * *)}\end{array}$ & - & 675,97 \\
\hline & \multicolumn{2}{|l|}{ TOTAL } & $1.815,00$ \\
\hline
\end{tabular}

(*) Se asume que se contrae infección al inicio de las relaciones sexuales, considerando la misma esperanza de vida que los no infectados;

(**) Precio medio de vacunar a una persona sana a lo largo de toda la vida excepto triple vírica y varicela; $(* * *)$ Precio medio de vacunar a una persona sana a lo largo de toda la vida.

\section{Tabla 4}

Coste de vacunar a lo largo de toda la vida en grupos de riesgo según vacuna: enfermedades crónicas.

\begin{tabular}{|c|c|c|c|}
\hline $\begin{array}{c}\text { Grupo } \\
\text { de riesgo }\end{array}$ & Vacunas & $\begin{array}{l}\text { Precic } \\
\text { dosis }\end{array}$ & $\begin{array}{c}\text { Coste final } \\
\text { con adminis- } \\
\text { tración }\end{array}$ \\
\hline \multirow{6}{*}{$\begin{array}{l}\text { Enfermedad } \\
\text { renal crónica } \\
\text { avanzada y } \\
\text { hemodialisis }\end{array}$} & Gripe anual & 2,90 & 178,00 \\
\hline & Neumococo 13 & 42,69 & 48,69 \\
\hline & Neumococo 23 & 10,2 & 16,2 \\
\hline & $\begin{array}{l}\text { Hepatitis B } \\
\text { diálisis / } \\
\text { prediálisis }\end{array}$ & 22,77 & 86,31 \\
\hline & $\begin{array}{l}\text { Vacunas } \\
\text { calendario }^{(* *)}\end{array}$ & - & 675,97 \\
\hline & \multicolumn{2}{|l|}{ TOTAL } & $1.005,17$ \\
\hline \multirow{6}{*}{$\begin{array}{l}\text { Enfermedad } \\
\text { hepática } \\
\text { crónica }\end{array}$} & Neumococo 23 & 10,2 & 16,2 \\
\hline & Hepatitis A & 29,95 & 71,90 \\
\hline & Hepatitis B & 7,64 & 40,92 \\
\hline & Gripe anual & 2,90 & 178,00 \\
\hline & $\begin{array}{l}\text { Vacunas } \\
\text { calendario }^{(* *)}\end{array}$ & - & 675,97 \\
\hline & \multicolumn{2}{|l|}{ TOTAL } & 982,99 \\
\hline
\end{tabular}

(*) Se asume que la condición se adquiere a los 50 años con una esperanza de vida media de 20 años; (**) Precio medio de vacunar a una persona sana a lo largo de toda la vida.

EPIRCE (Epidemiología de la insuficiencia renal crónica en España) realizado en mayores de 20 años, un $10 \%$ de la población adulta (3.300.000 personas) sufría de algún modo una insuficiencia renal crónica. En 2012, existían unos 20.000 pacientes en diálisis. Como en el resto de los análisis, la estimación de coste total depende del momento en el que se desarrolla la enfermedad, así como de la esperanza de vida media. El gasto estimado en la vacunación de una persona perteneciente a este grupo a lo largo de toda la vida fue de 1.005,17 euros.

- Enfermedad hepática crónica (EHC): se estima que 400.000 personas están afectadas con EHC en España, lo que equivale al 1,1\% de 


\begin{tabular}{|c|c|c|c|}
\hline \multicolumn{4}{|c|}{$\begin{array}{c}\text { Tabla } 5 \\
\text { Coste de vacunar a lo largo de toda la vida } \\
\text { en grupos de riesgo según vacuna: } \\
\text { trasplante de órganos. }\end{array}$} \\
\hline $\begin{array}{c}\text { Grupo } \\
\text { de riesgo }\end{array}$ & Vacunas & $\begin{array}{l}\text { Precio } \\
\text { dosis }\end{array}$ & $\begin{array}{l}\text { Coste final } \\
\text { con adminis- } \\
\text { tración }{ }^{(* * * *)}\end{array}$ \\
\hline \multirow{8}{*}{$\begin{array}{l}\text { Trasplante } \\
\text { de Órgano } \\
\text { Sólido }\end{array}$} & Gripe anual & 2,90 & 178,00 \\
\hline & Hepatitis A & 29,95 & 71,90 \\
\hline & Hepatitis B & 7,64 & 54,56 \\
\hline & Neumococo 13 & 42,69 & 48,69 \\
\hline & dTpa & 14,95 & 41,90 \\
\hline & Neumococo 23 & 10,2 & 16,20 \\
\hline & $\begin{array}{l}\text { Vacunas } \\
\text { calendario }^{(* *)}\end{array}$ & - & 675,97 \\
\hline & \multicolumn{2}{|l|}{ TOTAL } & $1.087,22$ \\
\hline \multirow{18}{*}{ TPH } & Neumococo 13 & 42,69 & 146,07 \\
\hline & Hexavalente & 29,95 & 107,85 \\
\hline & Neumococo 23 & 10,2 & \multirow{5}{*}{170,56} \\
\hline & Meningococo B & 62,95 & \\
\hline & $\begin{array}{l}\text { Meningococo } \\
\text { ACWY }\end{array}$ & 32,30 & \\
\hline & Hepatitis $\mathrm{A}^{(* * *)}$ & 29,95 & \\
\hline & VPH & 29,16 & \\
\hline & Hexavalente & 29,95 & \multirow{5}{*}{190,31} \\
\hline & Meningococo B & 62,95 & \\
\hline & $\begin{array}{l}\text { Meningococo } \\
\text { ACWY }\end{array}$ & 32,30 & \\
\hline & Hepatitis $\mathrm{A}^{(* * *)}$ & 29,95 & \\
\hline & VPH & 29,16 & \\
\hline & Triple Vírica & 7,10 & \multirow{2}{*}{66,10} \\
\hline & Varicela & 19,95 & \\
\hline & VPH & 29,16 & 35,16 \\
\hline & Gripe anual & 2,90 & 178,00 \\
\hline & $\begin{array}{l}\text { Vacunas } \\
\text { calendario }^{(* *)}\end{array}$ & - & 675,97 \\
\hline & \multicolumn{2}{|l|}{ TOTAL } & $1.570,02$ \\
\hline \multicolumn{4}{|c|}{$\begin{array}{l}(*) \text { Se asume que la condición se adquiere a los } 50 \\
\text { años con una esperanza de vida media de } 20 \text { años; } \\
(* *) \text { Precio medio de vacunar a una persona sana a lo } \\
\text { largo de toda la vida; }(* * *) \text { En caso de riesgo elevado de } \\
\text { exposición; }(* * * *) \text { Incluidas todas las dosis necesarias. } \\
\text { Se considera una sola administración si coincide en } \\
\text { el tiempo la administración de varias vacunas. }\end{array}$} \\
\hline
\end{tabular}

la población. Si consideramos 50 años de edad media al diagnóstico y 20 de esperanza de vida, a lo largo de toda la vida el coste previsto en la vacunación de una persona perteneciente a este grupo fue de 982,99 euros.

- Trasplante de órgano sólido: en España se realizaron 4.821 trasplantes de órgano sólido durante 2016. Con las premisas de 50 años de edad media al diagnóstico y 20 años de esperanza de vida, el coste estimado en la vacunación de una persona perteneciente a este grupo a lo largo de toda la vida fue de 1.098,90 euros.

- Trasplante de progenitores hematopoyéticos (TPH): en España se realizaron $3.068 \mathrm{TPH}$ durante el año 2015. Considerando el caso extremo de mayor coste, la vacunación a lo largo de toda la vida de una persona perteneciente a este grupo supuso 1.067,97 euros.

Coste total de vacunar a lo largo de toda la vida. La vacunación a lo largo de toda la vida en la población sana nacida anualmente, considerando los datos de 2018, supuso para la Administración un coste de 249.120.940,40 euros.

\section{DISCUSIÓN}

La vacunación es una de las medidas que más consiguen disminuir el impacto en la salud de las enfermedades infecciosas prevenibles por vacunación $^{(26)}$. Los programas de vacunación sistemáticos han conseguido disminuir en nuestro país la incidencia de enfermedades como la tosferina, la difteria, la poliomielitis, el sarampión, la rubeola, la parotiditis y el meningococo $\mathrm{C}$ en torno a un 99\%. La morbilidad y mortalidad anual evitada con la vacunación en estas enfermedades es muy importante: 300.000 casos y casi 40 fallecimientos en sarampión, 160.000 casos y 11 muertes en rubéola, 280.000 casos de parotiditis, más de 2.000 casos y 200 muertes de poliomielitis, en torno a 400 casos y 90 muertes de tétanos, 60.000 casos y 130 
muertes de tosferina, más de 27.000 casos y 140 defunciones por difteria, 300.000 casos de varicela, 800 casos y 50 muertes por meningitis C. Todo esto, sin contar con los costes sanitarios y las secuelas secundarias a estas enfermedades, que ya no se producen gracias a los programas de vacunación $^{(27,28)}$. En España, vacunar a lo largo de toda la vida a las personas sanas conlleva un gasto aproximado de 700 euros por persona. Además, se estima que vacunar a lo largo de toda la vida a las personas con las condiciones de riesgo que requieren mayor número de vacunas oscila entre 982,99 y 1.815 euros por persona, según el grupo de riesgo.

En España, el gasto sanitario público fue en 2017 de 68.483 millones de euros, lo que equivale a 1.472 euros por habitante ${ }^{(29)}$. El Sistema Nacional de Salud invierte en vacunación un $0,25 \%$ de su presupuesto sanitario anual. Otros países de nuestro entorno con mayores gastos en salud, cuyos calendarios difieren poco del nuestro, no invierten en vacunas más de un $0,5 \%{ }^{(30)}$. Sin embargo, el gasto realizado desde el SNS en medicamentos varía entre un $25 \%$ a un $30 \%$ del gasto sanitario total (el segundo del mundo y un $40 \%$ por encima del promedio europeo $)^{(31)}$. Sólo el gasto en fármacos antidiabéticos supone el $1,25 \%$ del gasto sanitario total ${ }^{(32)}$. Por otro lado, el coste medio de cada ingreso hospitalario es de 4.916 euros $^{(33)}$. Sin embargo, en un estudio europeo se estimó que el tratamiento de un caso de sarampión suponía entre 209 y 480 euros por caso, mientras que el coste de la vacunación y control del sarampión era entre 0,17 y 0,97 euros por persona, sin incluir los costes de la enfermedad a largo plazo $^{(34)}$. Toda esta información sugiere que vacunar a lo largo de toda la vida es una medida de prevención de bajo coste, si tenemos en cuenta la carga de enfermedad, muerte y costes indirectos que puede evitar.

En todo caso, la interpretación de los resultados debe realizarse considerando las limitaciones del estudio. En primer lugar, los cálculos se realizaron según el calendario vigente, por lo que los resultados únicamente reflejan un momento del tiempo, si bien el calendario es flexible y estas cifras pueden variar en función de las modificaciones que se vayan incorporando. En segundo lugar, no se incluyó el IVA, que puede fluctuar también a lo largo del tiempo, por lo que para la Administración sanitaria el coste es superior al estimado. Por último, se utilizaron los precios de los Acuerdos Marco, aunque los precios finales tras la negociación realizada en las comunidades autónomas pueden ser algo más bajos, sin contar con que existen otros gastos no contemplados, como son los de gestión o de promoción de los programas de vacunación.

Un estudio que evaluó el coste de la vacunación a lo largo de toda la vida en distintos países en Europa occidental, incluyendo España $^{(6)}$, obtuvo en sus resultados un coste superior al encontrado en este trabajo, con grandes diferencias en el coste total en hombres y mujeres (921 euros para hombres y 1.339 euros para mujeres). Esta discrepancia puede deberse a que los autores utilizaron como precios de referencia para España los que figuran en el Colegio Oficial de Farmacéuticos, que son superiores a los obtenidos por la Administración sanitaria en nuestro país ${ }^{(35,36)}$.

El cálculo para las personas de grupos de riesgos plantea varias dificultades inherentes a la variabilidad de los individuos que los conforman. La esperanza de vida media es difícil de estimar, ya que la edad de inicio de esa condición de riesgo varía y puede suponer que reciba una vacunación adicional a la recibida en el calendario o una única vacunación orientada al tipo de patología o condición subyacente ${ }^{(9)}$. Las vacunas recibidas también son variables, $\mathrm{y}$ sólo la gripe está incluida en casi todos ellos. Por todos estos motivos, y a pesar de que se han intentado estimar los casos más extremos en cuanto a coste, los resultados obtenidos en esta estimación económica deben ser tenidos 
en cuenta con precaución, pues dependen de las características individuales y de cada enfermedad específica de base.

En todo caso, el mayor coste económico corresponde a la vacunación de personas con asplenia, deficiencias del complemento y tratamiento con eculizumab, seguido de las personas con TPH. Aunque el número de personas en estos grupos de riesgo es muy reducido en comparación con la población general y pese a que el mayor coste en vacunación se centra en las personas sanas, el coste de no vacunar correctamente a estos grupos también puede llegar a ser muy alto, ya que los costes de la atención sanitaria en los grupos de riesgo es mayor que en la población sana y puede incrementarse por el padecimiento de enfermedades inmunoprevenibles ${ }^{(37,38)}$. Además, vacunar correctamente a las personas sanas también supone proteger indirectamente a las personas de grupos de riesgo que no están bien vacunadas o no pueden ser vacunadas $^{(39)}$. El mayor coste en los grupos de riesgo se destina a la vacuna de la gripe anual, la única incluida en prácticamente todos ellos. Sin embargo, es en la vacunación frente a la gripe de adultos pertenecientes a grupos de riesgo donde se han encontrado resultados mayores en cuanto ...en cuanto a coste-efectividad. ${ }^{(40)}$.

Finalmente, profundizar en este tipo de estudios de costes puede ser esencial para la toma de decisiones en política sanitaria y para la óptima asignación de los recursos disponibles, pudiendo incentivar políticas de evaluación sistemática en los programas de vacunación existentes, las cuales permitan acrecentar la correcta vacunación de las personas a lo largo de toda la vida. Por otro lado, y ante la existencia de movimientos contrarios a la vacunación que pueden ser una amenaza potencial para los programas de vacunación al aumentar la desconfianza sobre las vacunas, es importante disponer de estrategias de comunicación dirigidas a la población y a los decisores para dar a conocer el impacto en la salud de vacunar junto con el relativo bajo coste ${ }^{(41)}$, por lo que podrían ayudar a reforzar los programas de vacunación en nuestro país y concienciar de su considerable valor.

\section{BIBLIOGRAFÍA}

1. Andre FE, Booy R, Bock HL et al. Vaccination greatly reduces disease, disability, death and inequity worldwide. Bull World Health Organ. 2008;86(2):140-146

2. Rémy V, Zöllner Y, Heckman U. Vaccination: The cornerstone of an efficient healthcare system. J Market Access Health Policy 2015; 3:27041.

3. Largeron N, Lévy P, Wasem J, Bresse X. Role of vaccination in the sustainability of healthcare systems. J Mark Access Health Policy. 2015;3:10.3402/jmahp.v3.27043. Published 2015 Aug 12. doi:10.3402/jmahp.v3.27043

4. Consejo Interterritorial del Sistema Nacional de Salud. Ministerio de Sanidad, Consumo y Bienestar Social. Calendario común de vacunación a lo largo de toda la vida. Consultado el 25/05/2019. Disponible en: https:/www. mscbs.gob.es/profesionales/saludPublica/prevPromocion/ vacunaciones/docs/CalendarioVacunacion_Todalavida.pdf

5. Quilici S, Smith R, Signorelli C. Role of vaccination in economic growth. J Market Access Health Policy. 2015; 3:27044.

6. Grupo de Trabajo Criterios 2011, de la Ponencia de Programa y Registro de Vacunaciones. Criterios de evaluación para fundamentar modificaciones en el Programa de Vacunación en España. Comisión de Salud Pública del Consejo Interterritorial del Sistema Nacional de Salud. Ministerio de Sanidad, Política Social e Igualdad. 2011. Consultado el 24/05/2019. Disponible en: http://www. mscbs.gob.es/profesionales/saludPublica/prevPromocion/ vacunaciones/docs/Criterios_ProgramaVacunas.pdf.

7. Olivier Ethgen, Murielle Cornier, Emilie Chriv et al. The cost of vaccination throughout life: A western European overview. Human Vaccin Immunother 2016, VOL. 12, NO. 8, 2029-2037.

8. Drummond M, O'Brien B, Sculpher M, Stoddart G, Torrance G. Methods for the Economic Evaluation of 
Health Care Programmes. 3rd Edition. Oxford: Oxford Medical Publications; 2005.

9. Gimeno JA, Rubio S, Tamayo P. (2006): Economía de la salud: fundamentos. Díaz de Santos, Madrid.

10. Grupo de trabajo vacunación en población adulta y grupos de riesgo de la Ponencia de Programa y Registro de Vacunaciones. Vacunación en población adulta. Comisión de Salud Pública del Consejo Interterritorial del Sistema Nacional de Salud. Ministerio de Sanidad, Consumo y Bienestar Social, septiembre 2018. Disponible en: https:// www.mscbs.gob.es/profesionales/saludPublica/prevPromocion/vacunaciones/docs/Vacunacion_poblacion_adulta.pdf.

11. Grupo de trabajo vacunación en población adulta y grupos de riesgo de la Ponencia de Programa y Registro de Vacunaciones. Vacunación en grupos de riesgo de todas las edades y en determinadas situaciones. Comisión de Salud Pública del Consejo Interterritorial del Sistema Nacional de Salud. Ministerio de Sanidad, Consumo y Bienestar Social, julio 2018. Consultado el 24/05/2019. Disponible en: http://www.mscbs.gob.es/profesionales/saludPublica/ prevPromocion/vacunaciones/VacGruposRiesgo/docs/ VacGruposRiesgo_todas_las_edades.pdf.

12. Vallejo Torres L, Linertová R, Sanromá Ramos E, Ramos García V, Toledo Chávarri A, Herrera Ramos E, Pérez Martín JJ, Limia Sánchez A, Soler Soneira M, Castilla Catalán J, García Cenoz M, Tamames Gómez S, Imaz Iglesia I, Chalco JP, García Rojas A, Serrano-Aguilar PG. Coste-efectividad de la vacunación frente a herpes zóster. Ministerio de Sanidad, Consumo y Bienestar Social. Servicio de Evaluación del Servicio Canario de la Salud; 2018. Informes de Evaluación de Tecnologías Sanitarias.

13. Mokiou S, Standaert B, Li X, De Cock E. Measuring the cost of a pediatric vaccine administration in the UK. Vaccine. 2018 Jan 4;36(2):237-242.

14. OECD. State of Health in the EU España Perfil Sanitario del país 2017. Disponible en: https://ec.europa.eu/health/sites/health/files/state/docs/chp_es_spanish.pdf.
15. Van Walraven C, Manuel DG, Knoll G. Survival trends in ESRD patients compared with the general population in the United States. Am J Kidney Dis. 2014 Mar;63(3):491-9.

16. Zhang W, Sun B. Impact of age on the survival of patients with liver cancer: an analysis of 27,255 patients in the SEER database. Oncotarget. ;6(2):633-641.

17. Martin PJ, Counts GW Jr, Appelbaum FR et al. Life expectancy in patients surviving more than 5 years after hematopoietic cell transplantation. J Clin Oncol. 2010;28(6):1011-1016.

18. Boletín Oficial del Estado. Anuncio de formalización de contratos de: Subsecretaría de Sanidad, Servicios Sociales e Igualdad. Objeto: Acuerdo marco selección de suministradores de vacunas de calendario y otras para determinados órganos de contratación de AGE, las ciudades de Ceuta y Melilla y varias Comunidades Autónomas. Expediente: 201604AM0001. BOE núm. 102, de 29-04-2017.

19. Boletín Oficial del Estado. Anuncio de licitación de: Subsecretaría de Sanidad, Servicios Sociales e Igualdad. Objeto: Acuerdo Marco selección de suministradores de vacunas gripe estacional para determinados órganos de contratación de AGE, INGESA y las ciudades de Ceuta y Melilla y varias Comunidades Autónomas. Expediente: 201901AM0001. BOE núm. 211, de 2 de septiembre de 2017.

20. Área de Vigilancia de VIH y Comportamientos de Riesgo. Vigilancia Epidemiológica del VIH y sida en España 2016: Sistema de Información sobre Nuevos Diagnósticos de VIH y Registro Nacional de Casos de Sida. Plan Nacional sobre el Sida - S.G. de Promoción de la Salud y Epidemiología / Centro Nacional de Epidemiología - ISCIII. Madrid; Nov 2017. Consultado el 25/05/2019. Disponible en: https://www. mscbs.gob.es/ciudadanos/enfLesiones/enfTransmisibles/sida/ vigilancia/InformeVIH_SIDA_2017_NOV2017.pdf.

21. Milá Llambí J, Etxagibel Galdos A, Matamoros Florí N. The Spanish registry of primary immunodeficiencies (REDIP). Allergol Immunopathol (Madr) 2001; 29: 122-125. 
22. Otero A, de Francisco A, Gayoso P et al. Prevalence of chronic renal disease in Spain: Results of the EPIRCE study. Nefrología 2010; 30: 78-86.

23. Martínez-Castelao A, Górriz-Teruel JL, Bover-Sanjuán $\mathrm{J}$ et al. Detección y manejo de la ERC. Documento de consenso. Nefrologia (Madr.) 2014; 34: 243-262.

24. GBD 2015 Disease and Injury Incidence and Prevalence Collaborators. Global, regional, and national incidence, prevalence, and years lived with disability for 310 diseases and injuries, 1990-2015: a systematic analysis for the Global Burden of Disease Study 2015. Lancet 2016; 388: 1545-1602.

25. Organización Nacional de Trasplantes. Memoria ONT 2016. Consultado 25/05/2019. Disponible en: http://www.ont. es/infesp/Memorias/Memoria\%20Introducci\%C3\%B3n.pdf.

26. Organización Nacional de Trasplantes. Memoria Trasplante Progenitores Hematopoyéticos 2015. Disponible en: http://www.ont.es/infesp/Memorias/memoria\%20actividad\%20\%20tph\%202015.pdf. Consultado 25/05/2019.

27. Delany I, Rappuoli R, De Gregorio E Vaccines for the 21st century EMBO Mol Med. 2014 Jun; 6(6): 708-720.

28. Centro Nacional de Epidemiología. CIBER Epidemiología y Salud Pública (CIBERESP). Instituto de Salud Carlos III. Resultados de la vigilancia epidemiológica de las enfermedades transmisibles. Informe anual 2016. Madrid, 2018.

29. Pachón I. Historia de los programas de vacunación. En: Epidemiología de las enfermedades transmisibles incluidas en un programa de vacunación. Monografía de la Sociedad Española de Epidemiología; Madrid 2006 pp. 9-16.

30. Ministerio de Sanidad, Consumo y bienestar Social. Estadística de Gasto Sanitario Público 2017: Principales resultados. Marzo 2019. Consultado el 15/10/2019. Disponible en: https://www.mscbs.gob.es/estadEstudios/estadisticas/docs/ EGSP2008/egspPrincipalesResultados.pdf.
31. Ethgen O, Baron-Papillon F, Cornier M. How much money is spent on vaccines across Western European countries? Hum Vaccin Immunother. 2016 Aug 2;12(8):2038-2045.

32. Martínez J, López-Picado A, Latorre A, Apiñaniz A, Iturralde JM, Samper R, Marino A, Gil L. Evaluación de los factores relacionados con el gasto farmacéutico en atención primaria y la calidad de la prescripción en enfermedades crónicas. Investigación Comisionada. Vitoria-Gasteiz. Departamento de Salud, Gobierno Vasco, Vitoria- Gasteiz 2016. Informe Osteba D-16-05.

33. Crespo C, Brosa M, Soria-Juan A, López-Alba A, López-Martínez N, Soria B. Costes directos de la diabetes mellitus y de sus complicaciones en Espana (Estudio SECCAID: Spain estimated cost Ciberdem-Cabimer in Diabetes) Av Diabetol. 2013;29(6):182-189

34. Ministerio de Sanidad, Servicios Sociales e Igualdad. Subdirección General de Información Sanitaria e Innovación Dirección General de Salud Pública, Calidad e Innovación Indicadores Hospitalarios. Evolución 2010-2014. Consultado el 25/05/2019. Disponible en: http:/www.mscbs.gob.es/estadEstudios/estadisticas/estHospiInternado/inforAnual/documentos/IndicadoresHospitalarios2010_2014.pdf.

35. Carabin H, Edmunds WJ, Gyldmark M, Beutels P, Lévy-Bruhl D, Salo H, Griffiths UK. The cost of measles in industrialised countries. Vaccine. 2003 Oct 1;21(2730):4167-77.

36. Azzopardi-Muscat N, Schroder-Bäck P, Brand H. The European Union Joint Procurement Agreement for crossborder health threats: what is the potential for this new mechanism of health system collaboration? Health Econ Policy Law. 2017 Jan;12(1):43-59. Epub 2016 Jul 12.

37. Ministerio de Sanidad, Consumo y Bienestar Social. Notas de Prensa. 25 de noviembre de 2016. El Gobierno da luz verde al acuerdo marco que permite la compra centralizada de más de 20 millones de dosis de vacunas. Disponible en: https:// www.mscbs.gob.es/gl/gabinete/notasPrensa.do?id=4047. 
38. Pérez-Rubio A, Platero L, Eiros Bouza JM. Seasonal influenza in Spain: Clinical and economic burden and vaccination programmes. Med Clin (Barc). 2019 Jul 5;153(1):16-27.

39. Zhang D, Petigara T, Yang X. Clinical and economic burden of pneumococcal disease in US adults aged 19-64 years with chronic or immunocompromising diseases: an observational database study. BMC Infect Dis. 2018 Aug 29;18(1):436.

40. Kim TH, Johnstone J, Loeb M. Vaccine herd effect. Scand J Infect Dis. 2011;43(9):683-689. doi:10.3109/003 65548.2011.582247.
41. Ting EEK, Sander B, Ungar WJ. Systematic review of the cost-effectiveness of influenza immunization programs. Vaccine. 2017 Apr 4;35(15):1828-1843. doi: 10.1016/j.vaccine.2017.02.044.

42. European Centre for Disease Prevention and Control. Communication on immunisation - building trust. Stockholm: ECDC; 2012. 